\title{
Study of Motorized Spindle Reliability Monitoring
}

\author{
Hsun-Fu Chiang \\ Department of Mechanical Engineering \\ National Chung-Hsing University \\ Taichung, Taiwan \\ r43@mail.buffalo.com.tw \\ Jenq-Shyong Chen \\ Department of Mechanical Engineering \\ National Chung-Hsing University \\ Taichung, Taiwan \\ MichaelChen@dragon.nchu.edu.tw
}

\author{
Paul Chang \\ Department of Electrical Engineering \\ National Chang-Hua University of Education \\ Taichung, Taiwan \\ paulchang@mail.buffalo.com.tw \\ Chung-Yu Pan \\ Department and Institute of Industrial Engineering \\ and Enterprise Information \\ Tunghai University \\ Taichung, Taiwan \\ pancy@thu.edu.tw
}

\author{
Yi-Lin $\mathrm{He}$ \\ Buffalo Machinery CO., LTD. \\ Taichung, Taiwan \\ r47@mail.buffalo.com.tw
}

\begin{abstract}
This paper proposes an intelligent monitoring system to adapt motorized high speed spindle on machine tools to meet the requirement of Industries 4.0, based on the techniques of power electronic engineering, mechatronics and reliability engineering. In this paper, a force-sensor-integrated spindle is proposed to monitor the bearing stress during operation. The implementation of charge amplifier and mechanical design are described as well. As a result, raw data is transmitted through a filter to create fruitful information and allow the remote monitoring system to operate effectively. The information supports predictive maintenance plans and improves the quality of after-sales service.
\end{abstract}

Keywords: Intelligent Monitoring; Industries 4.0; Reliability Engineering

\section{INTRODUCTION}

A key factor in the most competitive manufacturing industries is the ability to manage complex industrial processes continuously. Different tasks are frequently performed by numerous venders in various geographical locations, which had been successfully achieved for several decades by employing information and communication technology (ICT). More than $90 \%$ of all industrial manufacturing processes are supported by ICT.

At present, powerful autonomous microcomputers are increasingly wirelessly networked with one another and covered by internet communication. This is resulting in the convergence of the physical and virtual world in the form of cyber-physical-systems (CPS). It is therefore possible to network resources, information, objects, and people to create the internet of things (IoT) and services, and this phenomenon will also have an effect on industry. In the manufacturing realm, this technological evolution can be described as the fourth-stage revolution. To achieve their goals, manufacturers expect high operational reliability and data link availability, which are crucial for mechanical and automation engineering. Guaranteed latency times and stable connections are thus key issues, since there have a direct impact on application performance. Furthermore it is beneficial to integrate the different established perspectives that currently exist in the realms of production, mechanical, process, and automation engineering, IT and the Internet, as well as knowledge and procedures of total quality management [1].

Leading manufacturers allow individual, customerspecific criteria to be included in the design, configuration, ordering, planning, manufacture, operation phases, and enable last-minute changes to be incorporated, such manufacturer need not be huge production plants. Flexible, CPS-based ad hoc networking enables dynamic configuration of different aspects of business processes. Furthermore, engineering processes can be made more agile, manufacturing processes can be changed, temporary shortages can be compensated for, and huge output increases can be achieved within a short time.

With optimistic decision-making, an update facility provides end-to-end transparency in real time, allowing early design decisions verification for engineering techniques. These are more flexible responses to disruption and global optimization across all sites of a company in the production area. Resource productivity and efficiency, the overarching strategic goals for industrial manufacturing processes, applying the highest possible production output from a given source volumes, and using the lowest possible amount of resources are important, to deliver a particular output for management. CPS enables manufacturing processes to be optimized on a case-by-case basis, 
across the entire value network, which is the aim of the industry 4.0 [2].

In term of supplying such a highly reliable production solution, the equipment produced needs to be of stable and reliable quality, and it should be possible to predict the Mean Time Between Failures (MTBF). Monitoring the complete set of equipment and its components becomes possible, and the production working conditions can be shared from management. A pre-check equipment warning message can provide enough time to the service team to ensure appropriate service and production remain on track.

As an equipment provider and machine tool supplier, it is extremely important to emphasize the use of the technical applications of IoT and data mining. This is to produce an intelligent machine for offering a product with predicable MTBF, a complete after-sales service plan, and real-time production, as well as provide analysis to support manufacturing management in creating the production program, input control, and output delivery timing. Furthermore, a full-time manufacturing management plan should be arranged.

In other words, when a product is operated in the field, regardless its location, the product manufacturer should be able to predict its life time and monitoring of machine working condition should be possible. In the case of a part deteriorating or before it stop working, it should be possible to set the time before failure occurs and the service team can arrange an appointment with the user. In this case, the service does not interfere with the original production plan. The user will be able to perform the production plan in optimization, ensuring that the user remain competitive and most profitable.

In Reliability and MTBF Overview-, speaks provides two categories for estimating the reliability of electronic equipment: (1) prediction based on individual failure rates, and (2) Demonstration based on operation of equipment over time. These prediction methods have several common assumptions, for example, constant failure rate, the use of thermal and stress acceleration factors, quality factors, and use conditions [3].

The high speed motorized spindle has been applied in various fields for decades, and monitoring of spindle thermal growth and vibration has been studied extensively. However, spindle life-time has not been studied much. High maintenance and long machine down time cost the user significantly, and spindle MTBF has become an important significant issue. Life-time monitoring provides valuable information to the user and equipment provider by offering a smart system to provide a service prior to its failure, save spindle down time, and minimize repair costs.

\section{INVESTIGATION OF SPINDLE FAILURE}

The motorized spindle has become the most popular tool when applying high-speed machining. Its performance indicates significantly improved the tolerance of fine machining, as well as fast cutting time, which cuts out a large percentage of energy usage, while high-speed cutting saves a lot of processing time. Furthermore, the machining parts are no longer required to move between different machines and set up fixtures from time to time, allowing for better performance. High-speed machining is one of the key factors in leading machining technology [4].

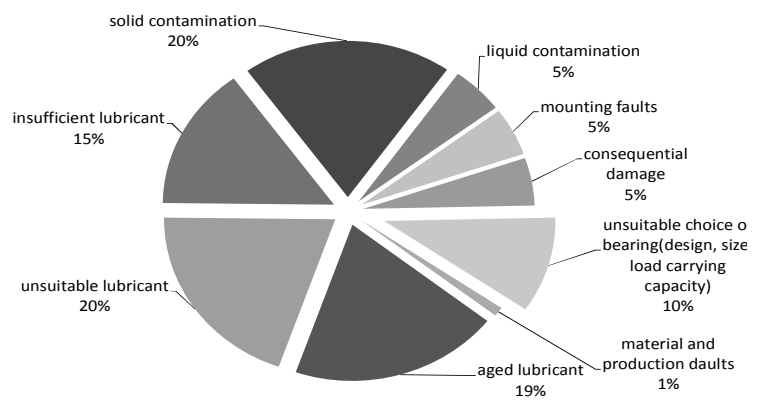

Figure 1. Bearing failure analysis [5]

High-speed machining provides significant benefits in cutting applications, particularly hard material machining. However, high-speed spindle is at high cost per unit, and damage may occur easily, due to lack of maintenance and unexpected collisions. The Fig. 1 shows the major reasons for bearing failure. The summary indicates that $80 \%$ of damage was result of external faults. It is therefore extremely important to ensure that the bearings always operate under normal condition, and a real-time monitoring system is essential. Moreover, the spindle design should be adapted to MTBF modes. The operator can always be certain about the spindle life, and the production cost is well considered in term of about wearing-out costs. The spindle is always active whenever the application is expected, in this way, a reliable spindle meets the requirement of a reliable device adapting to the requirements of the fourth industrial revolution (Industries 4.0).

Failure modes for bearings have been discussed, as indicated in the above figure. The modes are classified as fatigue, wear, correction, plastic deformation, fracture, and electrical erosion. The main causes of failure include loss of lubrication, incorrect mounting of bearing, misalignment, over- load, and excessive load. Since the external load, for example, cutting force on tool, acts directly on the bearing, bearing failure is strongly associated with these stresses [6]. Therefore, it is essential to consider the appropriate force acting on the spindle. In this paper, a systematic approach to the applicability of a given bearing configuration is discussed.

\section{MeChanicAl Profile of THE PIEZO-ELECTRIC SENSOR RING}

A real-time monitoring device is required for the high-speed spindle to ensure any abnormal bearings revolution conditions are detected. Some researchers have considered placing a vibration-detection unit outside the spindle, and although this works, it is not an optimal solution. The operator may need to be made aware once the abnormal bearing motion conditions. 


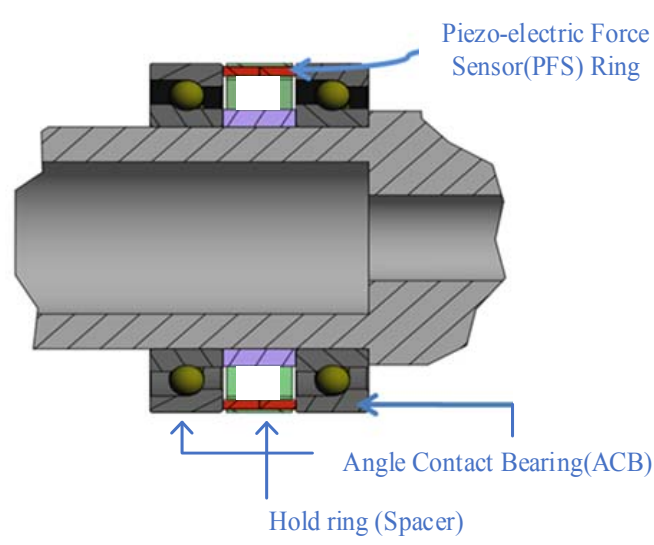

Figure 2. Spindle bearings with force sensor assembly

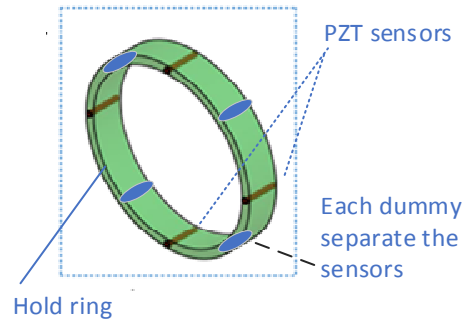

Figure 3. The proposed PFS ring design with holding ring and PZT sensor

A direct monitoring unit placed in front of the bearing has been proposed, as shown in Fig. 10. The sensing system includes the spindle bearing and sensor ring assembly, and the piezo electric sensor is located in the hold ring, as indicated in Fig. 2.

The Fig. 3 shows all the applied components, where are the PZT force sensors, dummies and holding ring. The holding ring placed between both angular contact bearings. The holding ring is also applied as a spacer between bearings. The PZT sensor is a highly sensitive sensor. The sensitivity is $-4 \mathrm{pC} / \mathrm{N}$. The separated angle is dependent with the number of the PZT sensors. If 4 sensors are adopted, the 4 pcs is placed by every 90 degrees of the ring, and both sides of the rings are provided with the same unit to enable a real-time monitoring function.

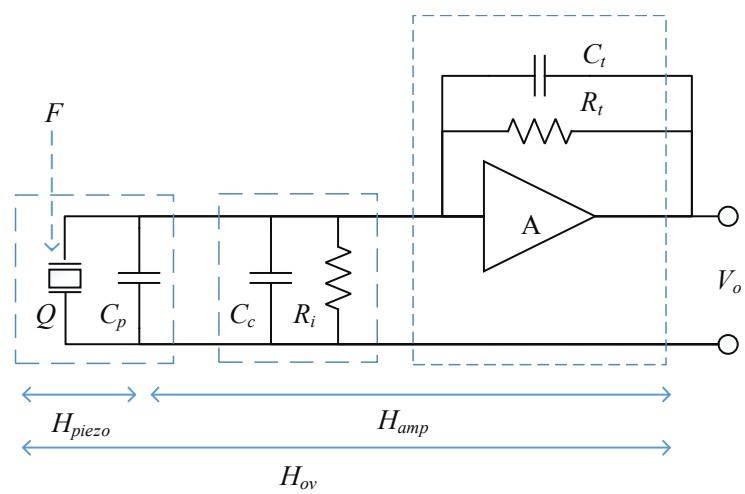

Figure 4. The simplified circuit diagram of charge amplifier with piezoelectric sensor

\section{The PIEZO-ELECTRIC SENSOR AND CHARge AMPLIFIER DESIGN}

The equivalent circuit of a piezoelectric sensor measuring system is illustrated in Fig. 4, which shows that the system includes a piezoelectric sensor, sensing cable, Charge Amplifier (CA) and Output Stage Amplifier (OSA). The cable loading effect is neglected, due to the unique properties of charge amplifier.

The gain of the overall system can be regarded as consisting of three stages, sensor, CA stage and OSA stage, with gains of $\mathrm{H}_{\text {piezo }}, \mathrm{H}_{\mathrm{amp}}$, and $\mathrm{H}_{\mathrm{OSA}}$, respectively. The gain of each stage is described in the following section.

\section{A. The PZT sensor gain $H_{\text {piezo }}$ and the charge/force property of piezoelectric sensor}

The property of piezoelectric material can be represented by the constitutive equation in the IEEE Standard on Piezoelectricity, 1987. ANSI/IEEE. Std. 176). The piezoelectric material includes two applications, sensor and actuator. In the sensor application, the external voltage field is zero, and the sensor is exposed to an external force. The constitutive equation can be written as $D=d^{d} \sigma$. Assume that there is one directional force acting on a stressed material with a cross-section area A, then the vector $\boldsymbol{D}$ of size $(1 / 3)$ can be simplified to a scalar equation, as shown in (1), which describes the relationship between stress and electric displacement. As the force stress is applied to the piezoelectric material, electric displacement is induced. Furthermore, total charge is described in (2), where the total charge amount is proportional to the electric displacement $\boldsymbol{D}$ and piezoelectric coefficient $d$.

$$
D=d \delta
$$

$$
Q=\int D d A
$$

where:

$D$ : Electric displacement [Coulomb $/ \mathrm{m}^{2}$ ]

$d$ : Piezoelectric coefficient, sensitivity of force to charge [Coulomb/Newton]

$\sigma$ : Stress acting on material $\left[\mathrm{Newton} / \mathrm{m}^{2}\right]$

$Q$ : Induced charge [Coulomb]

Combining (1) and (2), the overall force to charge response is represented as (3).

$$
Q=D d \sigma=d F
$$

Hence, in the first stage, the gain becomes

$$
H_{\text {piezo }}=Q / F=d,
$$

The sensor manufacturer's datasheet describes the piezoelectric coefficient $d$, for a typical piezoelectric sensor. Normally, the piezoelectric coefficient (or sensitivity) ranges is from several to tens of $\mathrm{pC}$. For example, Fig. 5 shows that the sensor sensitivity is -4 $\mathrm{pC} / \mathrm{N}$ over a wide measuring force range, and a linear 
feature meets the requirement of monitoring application.

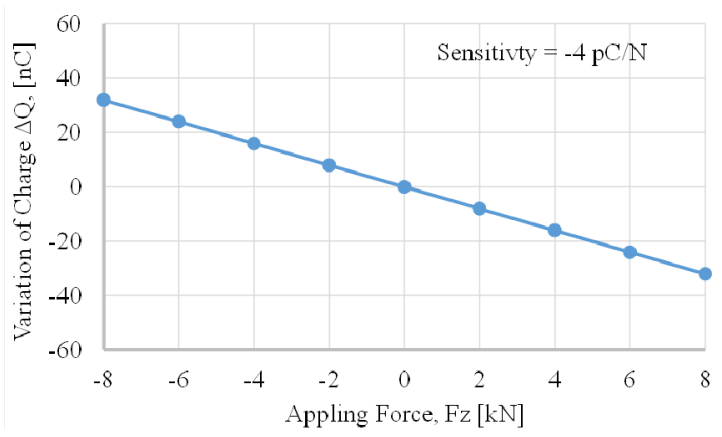

Figure 5. Characteristics of piezoelectric force sensor, an example for sensitivity of $-4 \mathrm{pC} / \mathrm{N}$. The commercial sensor from

\section{B. Signal conditioning of piezoelectric sensor $V / Q$}

Because the piezoelectric sensor has very high output impendence and the general measuring device only has input impendence in the order of several $\mathrm{M} \Omega$, the loading effect is an issue for piezoelectric measuring devices. For example, the input impendence of an oscilloscope and data recording device is $1 \mathrm{M} \Omega$. The charge amplifier offers an advantage in term of signal conditioning of the piezoelectric sensor. As mentioned previously, the stray capacitor of the cable is regardless.

In the second stage, the gain is

$$
H_{\text {amp }}(s)=V_{o} / Q=-s T_{t} /\left[C_{t}\left(1+s T_{t}\right)\right], T_{t}=R_{t} C_{t}
$$

In (5), the first term means that the voltage gain is dominated by the capacitor $\mathrm{C}_{\mathrm{t}}$. The circuit is a highpass filter, and in (5), once the measuring frequency is very close to quasi-static frequency, the output voltage of the circuit is eliminated to a significant small signal. The time constant $T_{t}$ enhances the $3 \mathrm{~dB}$ frequency to be as close as possible to quasi-static frequency, however, the large $C_{t}$ is significant for eliminating circuit sensitivity, which is the gain $\mathrm{H}_{\mathrm{amp}}$. In term of the time domain, as the charge $\mathrm{Q}$ is applied to the piezoelectric sensor, the output voltage of the amplifier decays with time. And it performs like a $\mathrm{R}_{\mathrm{t}} \mathrm{C}_{\mathrm{t}}$-circuit characteristic with a finite time constant, $T_{t}$. A larger resistance $R_{t}$ is effective to enhance the measurability of quasi-static frequency. However, at the end of a measuring cycle, the large time constant $T_{t}$ makes slowly the voltage decay on the capacitor. It is hardly to measure the other force input. To overcome the aforementioned problem, a reset switch M1 is connected in parallel to the charge amplifier circuit, as shown in Fig. 4, where $\mathrm{M} 1$ is used to reset the charge on the capacitor $\mathrm{C}_{\mathrm{t}}$. The coordination of an additional analogue/digital converter (ADC) and digital signal processing unit is required for data sampling, processing and digital/analogue filtering.

In the second stage of the signal condition, the circuit becomes where $\mathrm{H}_{\text {ins }}$ also represents the charge sensitivity of the CA. Notably, the frequency response term is shown in (6), and is based on the data sampling rate, performance of processing unit, and bandwidth of the external digital/analogue filter.

\section{Voltage gain of overall circuit}

The voltage gain of the third stage is dependent on the amplifier design. Normally, this stage can be inverted or non-inverted amplifier. (7).

The voltage gain of the circuit can be written as

$$
H_{o}(s)=H_{\text {piezo }} H_{\text {amp }} H_{O S A}
$$

\section{EXPERIMENT RESULT}

\section{A. The Prototype and Sensor Testing}

The adopted PZT is shown in Table 1. The implemented PFS ring is shown in Fig. 6 at the right side. The relationship of the generated charge and force for four sensors are shows in Fig. 7. The sensitivity for four piezo electric sensors is illustrated in Table 2. It shown that the sensitivity is closing 4 $\mathrm{pC} / \mathrm{N}$. The phenomenon of the non-linearity and hysteresis is observed in Fig. 6 within $1 \%$ non-ideal error.

\section{B. The Bandwidth Validation of Charge Amplifier}

The Bode plot diagram is shown in Fig. 8. The results shown the higher capacitance $\mathrm{Ct}$ is, the lower 3 $\mathrm{dB}$ frequency is. As the capacitance is increased with ten times for the three testing, the gain is decreased with $20 \mathrm{~dB}$. However, the low $3 \mathrm{~dB}$ frequency is lower such that the quasi-static measurement is possible. For the $100 \mathrm{nF}$ capacitance, the low $3 \mathrm{~dB}$ frequency is close to $1 \mathrm{mHz}$ and $10 \mathrm{mHz}$. To measure the bearing vibration is possible. At the testing signal frequency 1 $\mathrm{mHz}$, the phase is leading to the testing signal. As shown in Fig. 9 (b), the frequency is $100 \mathrm{mHz}$, the input and output signal is almost in phase. The output voltage of the charge amplifier is shown in Fig. 9.

TABLE I. The Parameter of Piezo Electric Sensor

\begin{tabular}{|c|l|}
\hline Sensitivity [pC/N] & $-4 \mathrm{pC} / \mathrm{N}$ \\
\hline Measurable normal force, $\boldsymbol{F}_{\boldsymbol{N}}[\boldsymbol{N}]$ & $3 \mathrm{kN}$ \\
\hline Measurable operating force $[\boldsymbol{N}]$ & $\mathrm{F}_{\mathrm{N}} * 115 \%$ \\
\hline Breaking force $[\boldsymbol{N}]$ & $\mathrm{F}_{\mathrm{N}} * 200 \%$ \\
\hline Sensor operation temperature $\left[{ }^{\circ} \boldsymbol{C}\right]$ & $-20 \sim 120$ \\
\hline Sensor storage temperature $\left[{ }^{\circ} \boldsymbol{C}\right]$ & $-20 \sim 120$ \\
\hline Fundamental resonance frequency $[\mathbf{H z}]$ & $105 \mathrm{k}$ \\
\hline
\end{tabular}

TABLE II. The SEnSitivity of Piezo Electric Sensor

\begin{tabular}{|c|l|l|l|}
\hline Position & $\begin{array}{c}\text { Sensitivity } \\
{[\mathbf{p C} / \mathbf{N}]}\end{array}$ & $\begin{array}{c}\text { Linearity } \\
{[\% \text { FSO] }}\end{array}$ & $\begin{array}{c}\text { Hysteresis } \\
{[\% \text { FSO] }}\end{array}$ \\
\hline$\# \boldsymbol{1}$ & 4.026 & 0.443 & 0.388 \\
\hline$\# \mathbf{2}$ & 3.998 & 0.539 & 0.335 \\
\hline$\# \mathbf{3}$ & 3.960 & 0.518 & 0.332 \\
\hline$\# \mathbf{4}$ & 4.074 & 0.455 & 0.253 \\
\hline
\end{tabular}

$$
H_{\text {amp }}(s)=V_{o} / Q=H_{\text {ins }}
$$




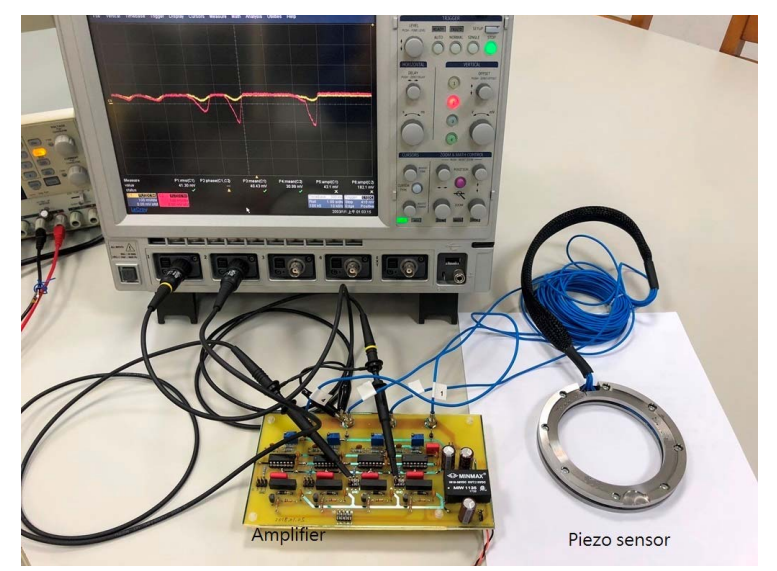

Figure 6. Prototype photograph

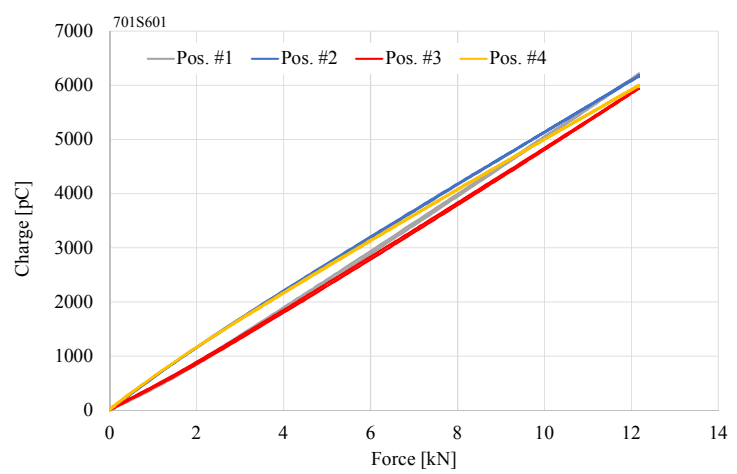

Figure 7. The testing for each piezo electric sensor

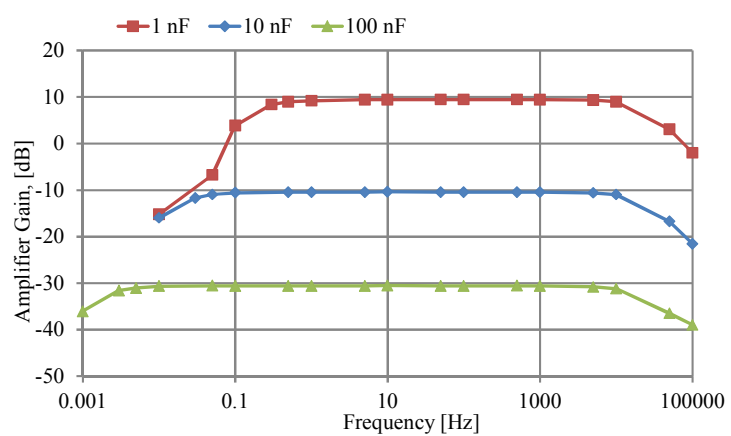

Figure 8. The Bode plot diagram - serval RC time-constant testing for the implemented charge amplifier

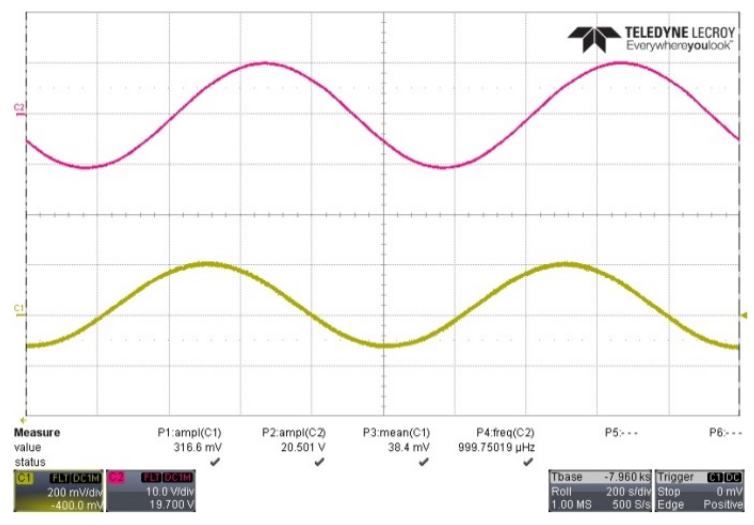

(a) $1 \mathrm{mHz}$

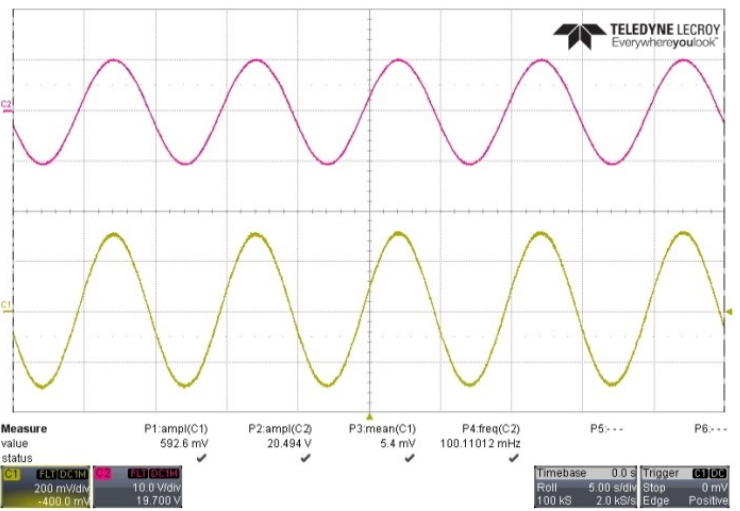

(b) $100 \mathrm{mHz}$

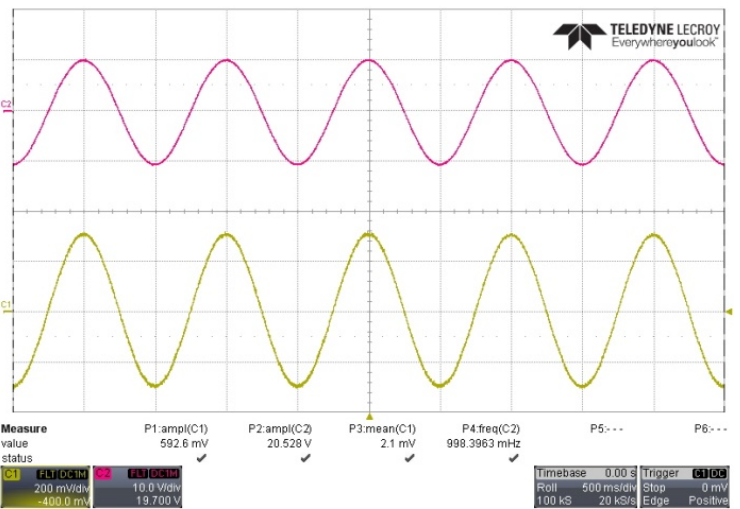

(c) $1 \mathrm{~Hz}$

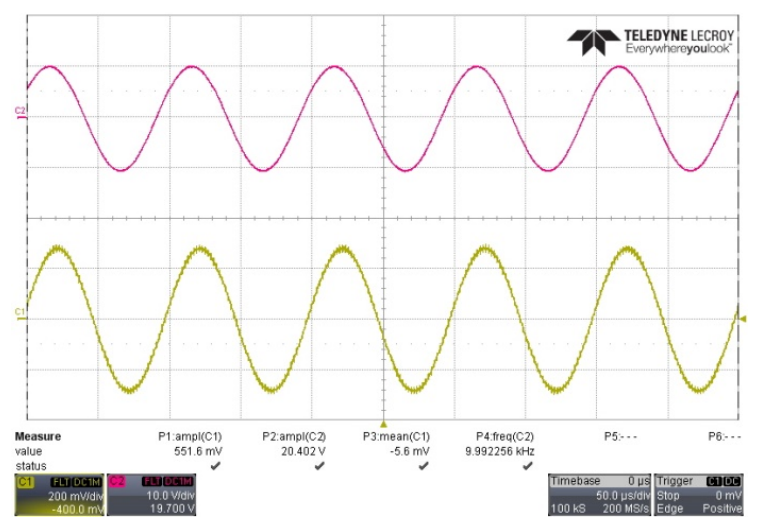

(d) $10 \mathrm{kHz}$

Figure 9. The experiment waveforms of the implemented charge amplifier - $\mathrm{Ct}=100 \mathrm{nF}$ 
Fig. 10 shows the measuring result of CA circuit, whereas a linear output is obtained, if it is confirmed that the CA produces a perfect output. The crossover phenomenon for the mechanical force link is observed. As shown in Fig. 11, it is tested via acting the force on Pos. \#1, Pos. \#2 and one dummy position between Pos. \#1 and Pos. \#2 by sequent. As the surface of PFS ring is well-contacted to the surface of $\mathrm{ACB}$, the identical force are applied on each dummies position and PZT position.

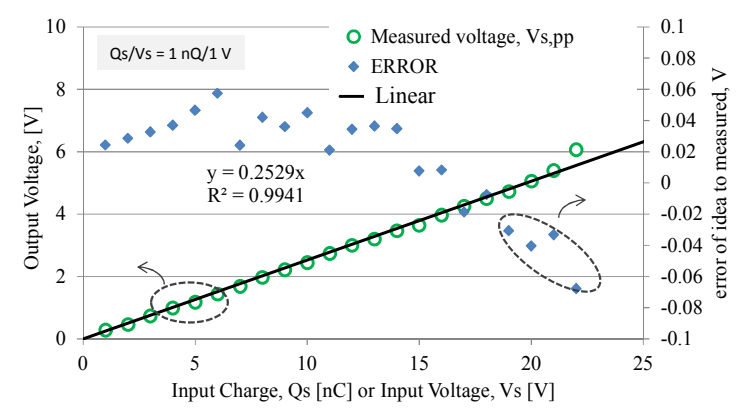

Figure 10. Linearity of Charge amplifier

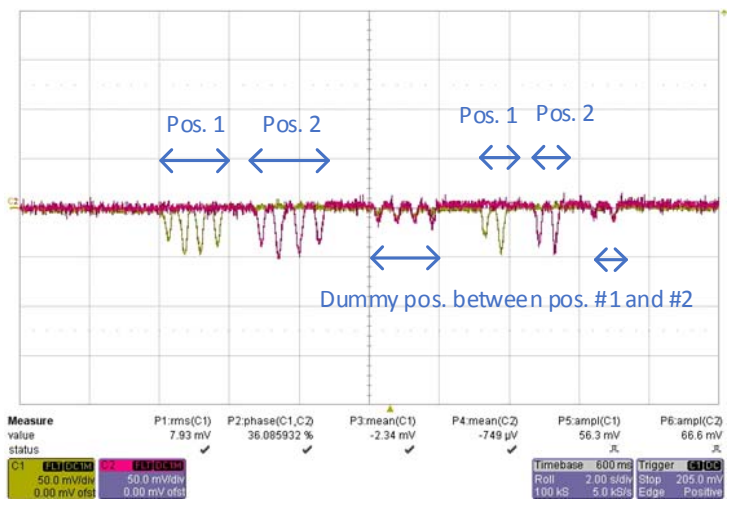

Figure 11. Force-link crossover phenomenon

\section{CONCLUSION}

Bearing treatment is considered to be major factor of spindle life, and a bearing set-up study. In this paper, a direct monitoring system is developed, and the simulation confirmed its functioning, while the sensing design makes the directly monitoring become possible. The aim of this research is to support the requirements for any high-speed spindle to adapting the requirement of the fourth industrial revolution equipment. Prior notice is becoming available, which can provide information before failure occurs. A perfect after-sales service can be provided instead of the equipment remaining dead for a few days to months, thereby meeting the requirements for Industries 4.0.

\section{ACKNOWLEDGMENT}

The authors would like to thank the Ministry of Economic Affairs of Taiwan, R.O.C., MOEA Taiwan supported this work under grant number MOEA 1042222-I-152-001.

\section{REFERENCES}

[1] Guide of Total Quality Management

[2] Industries 4.0 Working Group, "Recommendation for Implementing the Strategy Initiative Industries 4.0, Germany, Apr 2013.

[3] S Scott, "Reliability and MTBF Overview" Victor Reliability Engineering, May 2012.

[4] C.F. Chang and J.J. Chen "Vibration Monitoring of motorized spindles using spectral analyst techniques", in Mechatronics, Vol. 19, Issue 5, 2009

[5] FAG Group, (2005). Rolling Bearing Failure Analysis, FAG Australia Pty Ltd, 2005.

[6] SKF Group,. Bearing damage and failure analysis, Mar 2014

[7] Kistler, Instruction Manual of Charge Meter: charge meter type 5015A, 2017. 